\title{
STANDING-WAVE THERMOACOUSTIC ENGINES
}

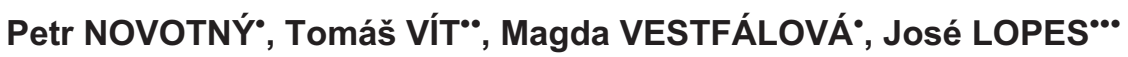

\begin{abstract}
The aim of this study is mainly devoted to explain a thermoacoustic effect. We explore the basic principles of thermoacoustic engines and primarily we focus on a quarter-wavelength closed-open thermoacoustic engine as a prime mover, where temperature difference imposed along the stack can lead to sound generation, acoustic work. Elementary standing wave theory is discussed, as well as the connection between thermodynamics and acoustical oscillations. Influence of the characteristic pore dimension in the stack as an important parameter in the design of thermoacoustic devices is reported. Investigation through this paper will be the basic instrumental apparatus for continuing research into the experimental and numerical study of the quarter-wavelength thermoacoustic engine.
\end{abstract}

\section{INTRODUCTION TO THERMOACOUSTIC}

The phenomena of thermoacoustic effects which convert heat energy to sound, acoustic work, have been known for over two hundred years. In the $19^{\text {th }}$ century Laplace established the adiabatic expansions and compressions of the sound wave associated with variations in temperature of the gas, Newton had assumed that the motion of sound wave of the gas is isothermally. These thermal effects in pressure and motion oscillations of sound wave in the gas are fundamental for the operation of thermoacoustic engines (prime movers) and refrigerators (heat pumps) as show in Figure 1.

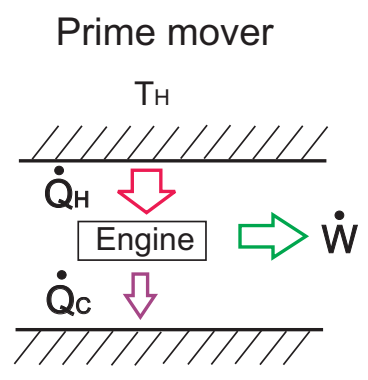

$\mathrm{Tc}$

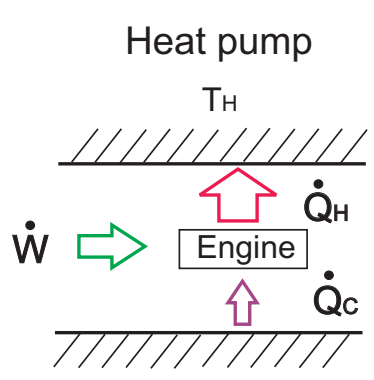

$\mathrm{TC}$

Figure 1: The principle of the prime mover (engine) and the heat pump (refrigerator)

\footnotetext{
- Ing. Petr Novotný: Technical University of Liberec; Studentská 2; 461 17, Liberec; CZ, e-mail: novotnypck@seznam.cz

•- doc. Ing. Tomáš Vít, Ph.D.: Toptec - Institute of Plasma Physics AS CR, v.v.i.; Skálova 89;

511 01, Turnov; CZ, e-mail: vit@ipp.cas.cz

•.• José Pedro Rodrigues Lopes: University of Minho, Campus de Gualtar, 4710-057 Braga, Portugal, e-mail: a53374@alunos.uminho.pt
}

This is an Open Access article distributed under the terms of the Creative Commons Attribution License 2.0, which permits unrestricted use, distribution, and reproduction in any medium, provided the original work is properly cited. 
In a prime mover, heat flows through the engine from high (a heat source) to low (a heat sink) temperature, and the engine generates an acoustic work. In a heat pump, the acoustic work of the actuator is absorbed by the engine and a heat is pumped from low temperature to high temperature by force of this work. In the insulation system the first law of thermodynamics is respected [1]:

$$
\dot{Q}_{H}-\dot{Q}_{C}-\dot{W}=0 .
$$

Rayleigh understood that oscillatory thermal expansion and contraction of the gas could create acoustic power: "If heat be given to the air at the moment of greatest condensation, or be taken from it at the moment of greatest rarefaction, the vibration is encouraged." [5]

These effects could be realized in the gas close to a solid surface of the narrow channel, where heat can be extracted or supplied to the gas from the surface. The results of coupled pressure and displacement oscillations (also temperature as a response of the pressure variations) in interaction of a heat transfer between the gas and the solid surface is that a sound wave is sustained in case of a large temperature gradient along the surface. While in the reserve case an acoustic work is absorbed in order to transport heat, generating a temperature gradient [7]. During the 1980 's and 1990 's Rott and coworkers from Los Alamos National Laboratory performed an accurate quantitative discussion on the thermoacoustic in a series of papers [6].

\section{BASIC PRINCIPLE OF STANDING-WAVE THERMOACOUSTIC ENGINE}

\subsection{A QUARTER-WAVELENGTH CLOSED-OPEN RESONATOR}

A travelling wave trapped in an enclosure at resonant frequency will result in a standing longitudinal wave in an air column. In our case we suppose a quarter-wavelength $(L=\lambda / 4)$ closed-open resonator where a node of pressure with a zero displacement is situated on the close end of the resonator (no phase change: the wave encounters with a greater acoustic impedance) and an antinode of pressure with a maximum displacement is situated on the open end to the surrounding ( $180^{\circ}$ phase change: the wave encounters with a lesser acoustic impedance) [11] which is shown in Figure 2 . The blue transitional background represents the actual arrangement of the molecules. The equation for the frequency of a standing wave travelling through the closed-open tube is given by [7]:

$$
f=\frac{a}{4 \cdot L},
$$

where $f$ is a resonant frequency, $a$ is velocity of the wave (a speed of sound) and $L$ is the length of the resonator. For example, in the resonator with length of $300 \mathrm{~mm}$ where the air with temperature $30^{\circ} \mathrm{C}$ is used as working fluid is the frequency of a standing wave $291 \mathrm{~Hz}$

When the stack is placed parallel to the direction of vibrating particles (somewhere between the pressure node and antinode, where an optimized temperature gradient can be generated in the stack) the initially isothermal plate will experience a temperature gradient gradually. In reverse of that, the imposed temperature gradient may induce particle oscillations [8]. 


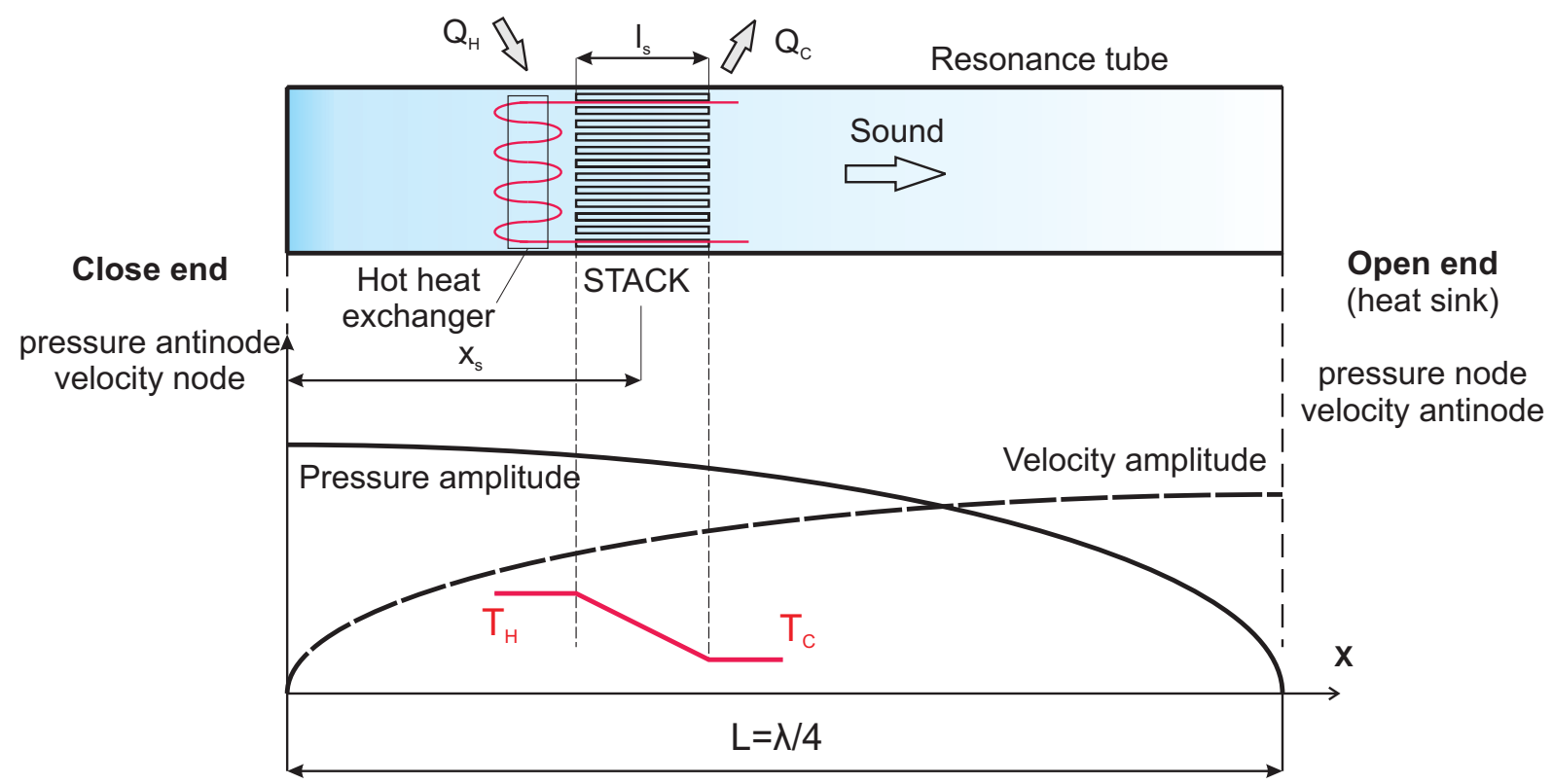

Figure 2: Illustration of the relationship between the pressure $(-)$, the phase of the sound wave (--) and the blue transitional background represents the actual arrangement of the molecules in the quarter-wavelength $(L=\lambda / 4)$ closed-open resonator.

In a pure standing wave pressure and displacement oscillations are in phase. We consider the uniform temperature throughout the entire resonator $T_{m}$. The parcel does not exchange heat with the outside $(\Delta s=0)$. The temperature $T_{1}$ and volume variations $V_{1}$ are exactly in and $180^{\circ}$ out of phase with the acoustic pressure $p_{1}$. The acoustic pressure $p_{1}$ and particle velocity $u_{1}$ are still $90^{\circ}$ out of phase. The ideal gas is assumed $\left(\beta \cdot T_{m}=1\right)[3]:$

$$
\begin{gathered}
T_{1}=\frac{\beta \cdot T_{m}}{\rho_{m} \cdot c_{p}} \cdot p_{1}=\frac{\kappa-1}{\kappa} \frac{T_{m}}{p_{m}} \cdot p_{1}, \\
V_{1}=-\kappa \cdot \frac{V_{m}}{p_{m}} \cdot p_{1} .
\end{gathered}
$$

Where $\kappa=c_{P} / c_{v}$ is the adiabatic coefficient of an ideal gas, $\beta$ is the isobaric volumetric expansion coefficient, $c_{p}$ and $c_{v}$ are the isobaric and the isochoric specific heat capacity and the subscripts $m$ refer to the mean properties of the value.

\subsection{BASIC PRINCIPLE OF THE THERMOACOUSTIC EFFECT}

The cycle of thermoacoustic heat engine can be realizing in the stack which is commonly made from a porous matter or parallel plates (is called the stack).

The prime-mover cycle also consists of two adiabatic (1-2, 3-4) and two isobaric heattransfer steps $(2-3,4-1)$ as a Brayton cycle. We apply a large temperature gradient $\left(\nabla T_{m}\right)$ along the channel. When a parcel of gas is compressed (1-2), it warms up but is still colder than the local wall position. The heat then flows from the wall into the parcel which expands (2-3). As a consequence of this expansion a gas (3-4) will be displaced to the other side of the stack. In step (4-1) a parcel of gas is warmer than the wall and the heat flows into the wall which is illustrated in Figure 3. A gas contracts and work is done by the acoustic work [7]. 


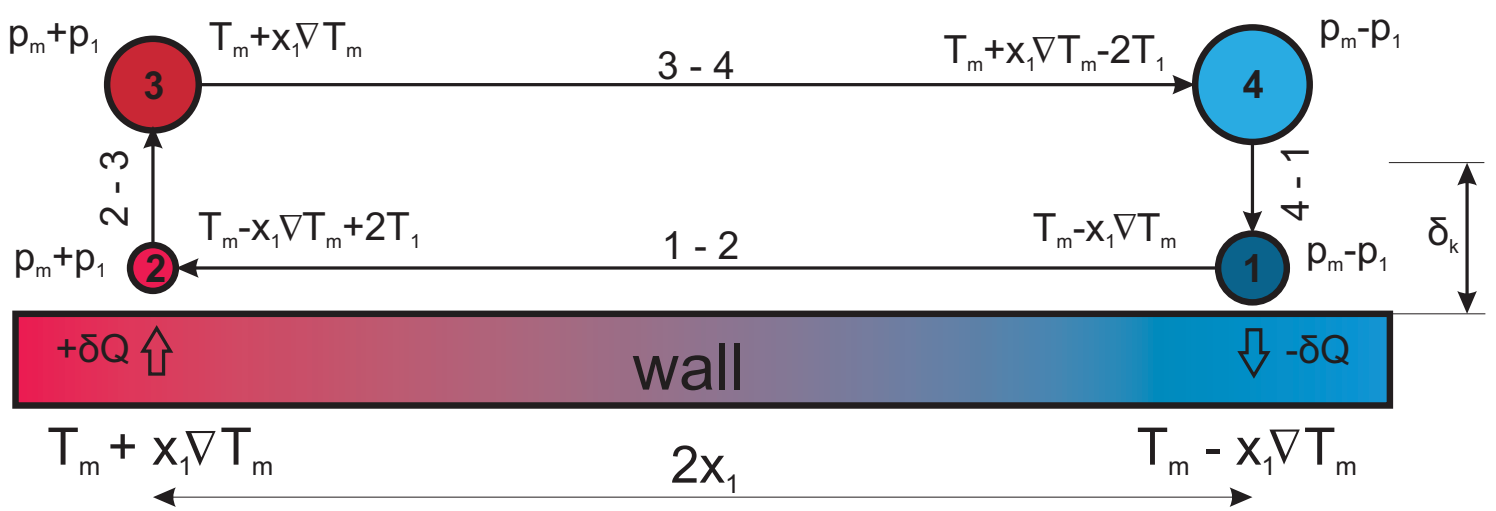

Figure 3: The parcel of gas which is neither isothermal nor adiabatic has imperfect thermal contact with the wall (a macroscopic Lagrangian view).

The only difference with the refrigerator case is that a small temperature gradient is generated along the stack by the acoustic work, so that the directions of heat transfer in steps 3-2 and 1-4 are reversed.

The net work produced in one cycle is given by the area 1234 in Figure 4 as a result of standing wave can be sustained by a large temperature gradient along the walls of the channel.


Figure 4: Schematic $p-V$ and $T-s$ diagram of the ideal thermoacoustic cycle.

1-2 adiabatic compression;

2-3 isobaric heat transfer;

3-4 adiabatic expansion;

4-1 isobaric heat transfer

\section{THE THERMAL PENETRATION DEPTH}

A parcel of gas in a narrow region has imperfect thermal contact with the wall. This affected zone at the edge of the thermal boundary layer which is coated the stack wall. The thickness of this layer depends on the frequency of standing wave $f$, the thermal conductivity $k$, density $\rho_{m}$ and the specific heat per unit mass at constant pressure $c_{p}$ of the gas that a heat can diffuse through the gas during the time $t=1 / f$. The thermal penetration depth is defined as [3]:

$$
\delta_{k}=\sqrt{\frac{k}{\pi \cdot \rho_{m} \cdot c_{p}}} \cdot \sqrt{\frac{1}{f}} .
$$


Gas parcels well outside and well inside the boundary layer are considered adiabatic and isothermal, respectively. In designing of the stack the optimal stack layer separation is four thermal penetration depths [1].

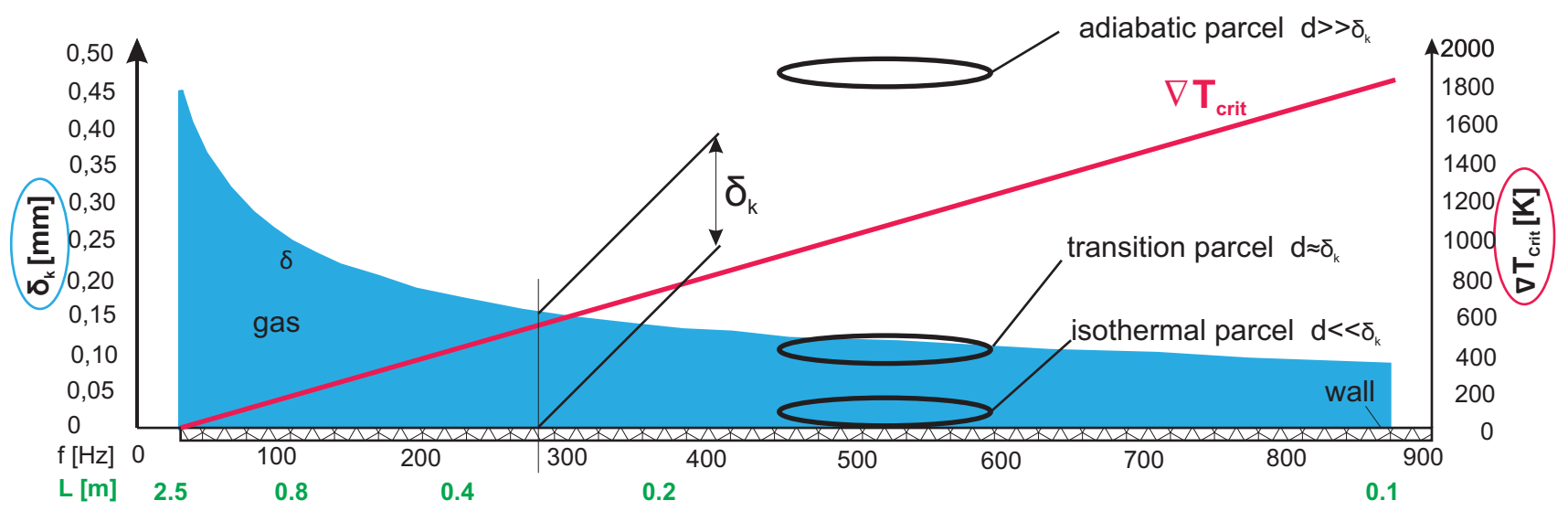

Figure 5: The dependence of the thermal boundary layer thickness and the critical temperature gradient (red line) on the frequency of the acoustic wave and on the length of resonator (air temperature is $30^{\circ} \mathrm{C}$ )

The elements of the gas far away from the wall $y \gg \delta_{k}$ have no thermal contact will expand and compress adiabatically and no heat transfer will take place. Elements that are too close to the wall $y \ll \delta_{k}$ have a good thermal contact the expansion and heat exchange will take simultaneously. Elements at about a distance of a thermal penetration depth $y \approx \delta_{k}$ from the wall have imperfect thermal contact to exchange some heat with the wall and produce a time delay motion and the heat transfer is necessary for the process.

\subsection{STACK}

The stack forms the heart of the thermoacoustic engines. The most common stack geometries have a characteristic constant cross-section channel along the direction of the flow. In standing-wave systems is beneficial to have pores with a radius of one or more thermal penetration depths, it is $N_{L} \geq 1$. This is necessary to create the optimal phasing between pressure and velocity and create an optimal heat-shutting effect. The highest acoustic power is obtained if $N_{L} \sim 1$, the Lautrec number is defined as [4]:

$$
N_{L}=r_{h} / \delta_{k}
$$

where a hydraulic radius $r_{h}=V_{G} / A_{W}$. is the ratio of the volume of the pores $V_{G}$ to the wetted area of the porous solid $A_{W}$. For the square section of the pore in stack the hydraulic radius can be expressed as the ratio of the pore 's cross-sectional area $A$ to its perimeter $\Pi: r_{h}=A / \Pi$. If $N_{L} \geq 1$, the porous medium is called a "stack". If $N_{L}<1$, the porous medium is called a "regenerator". The Lautrec number can indicate when your regenerator is "too loose". Four thermal penetration depths is the optimal stack layer separation for the edge of square section pore $b_{o p t}=4 \cdot \delta_{k}[1]$, [4]. 
To reduce the negative effect on the performance a material of the stack must be chosen with a low thermal conductivity. On the other hand, we must take a heat capacity $C_{S}$ larger than the heat capacity $C_{p}$ of the working gas, so that the plate temperature can be considered steady [10].

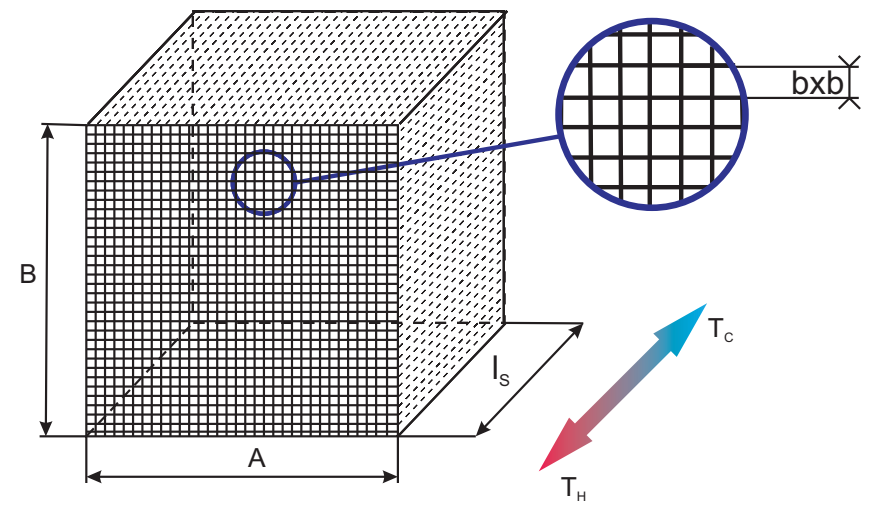

Figure 6: Illustration of the stack with square section of the pore

\subsection{THE VISCOUS BOUNDARY LAYER}

The above expression is derived for the ideal situation when no viscosity, the infinite heat capacity of the stack material and zero thermal conductivity of the gas and the solid in the longitudinal direction are considered. In real conditions these effects decrease overall efficiency of an engine. Similar to the thermal boundary layer there is a thinner viscous boundary layer defined as [3]:

$$
\delta_{v}=\sqrt{\frac{\eta}{\pi \cdot \rho_{m}}} \cdot \sqrt{\frac{1}{f}},
$$

with $\eta=v \cdot \rho_{m}$ being the shear viscosity where $v$ is the kinematic viscosity. The thermal and viscous penetration depths ratio are related by the Prandtl number, describes the extension of the thermoacoustic effects expected from any chosen working fluid:

$$
\sigma=\left(\frac{\delta_{v}}{\delta_{k}}\right)^{2}=\frac{\rho_{m} \cdot c_{p} \cdot v}{k} .
$$

A lower value for Prandtl number, characteristic of the inert gases, promotes thermoacoustic effects. For most gases (as air) is $\sigma \cong 2 / 3$. A mixture of the inert gases has also been used. For a mixture of Helium and Xenon $\sigma \doteq 0.2$ the Prandtl number can be that small [3], [4].

\subsection{THE LONGITUDINAL TEMPERATURE GRADIENT}

Thermoacoustic engines contain a porous ceramic stack, with the low thermal conductivity, sandwiched between two heat exchangers. The heat from the high temperature exchanger is supplied and on the other is withdrawn from the system at near ambient temperature. This temperature gradient across the stack is result of gas amplification of pressure disturbances and result in high levels of sound emitted once a steady state has been achieved.

This temperature has to be larger than critical temperature gradient for a thermoacoustic engines. The temperature gradient has to be steep enough so that the gas is colder than the wall during adiabatic compression at the peak displacement (the gas will receive 
energy from the wall) and warmer than the wall at the end of expansion at the other peak of displacement (the gas will give off heat energy to the wall).

Generally, the temperature of the gas as a function of the distance $y$, perpendicular to the wall is considered [1]:

$$
T_{1}(y)=\left(\frac{T_{m} \cdot \beta}{\rho_{m} \cdot c_{P}} \cdot p_{1}-\frac{\nabla T_{m}}{\omega} \cdot u_{1}\right) \cdot\left(1-e^{(i-1) \cdot y / \delta_{k}}\right) .
$$

The first term here is the temperature variation caused by adiabatic compressions and expansion of the gas $2 \cdot p_{1} / \rho_{m} \cdot c_{P}$. The second term represents the difference wall temperature $2 \cdot \xi_{1} \cdot \nabla T_{\text {crit }}$ over the maximum displacement of the gas molecules. Where $p_{1}$ and $\xi_{1}=u_{1} / \omega$ are an acoustic pressure and a displacement amplitudes, respectively. The fluid far from the wall $y \gg \delta_{k}$ when there is no thermal contact between the gas and the wall, the actual temperature oscillations are just a linear superposition or these two effects, than brackets with terms equals zero, we note that there is the critical temperature gradient which was derived by Swift and is given [1], [4]:

$$
\nabla T_{\text {crit }}=\frac{\omega \cdot p_{1}}{\rho_{m} \cdot c_{p} \cdot u_{1}} \cong \frac{2 \cdot T_{1}}{2 \cdot \xi_{1}}
$$

The critical temperature gradient depends on the operating angular frequency $\omega$ and the first order pressure $p_{1}$ and velocity $u_{1}$ in the standing wave as well as the mean density of gas $\rho_{m}$ and the isobaric specific heat $c_{p}$. If the wall temperature gradient is lower than critical one heat flows in the opposite direction from the gas into the hot reservoir and from the cold reservoir into the gas. This critical gradient sets the limit between the device function as a prime-mover or as a refrigerator.

\subsubsection{THE CRITICAL TEMPERATURE GRADIENT AS A SimpLE FUNCTION OF THE LENGTH OF the RESONATOR}

In the Equation 10 a ratio $Z=p_{1} / u_{1}$ is the characteristic impedance of a medium and it is a material property $Z=\rho_{m} \cdot a$, where $a$ is the longitudinal wave speed or a sound of speed in the ideal gas $a=\sqrt{\kappa \cdot p_{m} / \rho_{m}}$. The operating angular frequency counted with Equation $2 \omega=2 \cdot \pi \cdot a / 4 \cdot L$. The equation of the critical temperature gradient (10) combined with previous relations give a relation for $\nabla T_{\text {crit }}$ as a function of the length of the closed-open resonator $L$ (is depicted in Figure 5 ) and the thermodynamic properties of the working fluid:

$$
\nabla T_{\text {crit }}=\frac{\pi}{2} \cdot \frac{\kappa \cdot p_{m}}{c_{p} \cdot \rho_{m}} \cdot \frac{1}{L}=\frac{\pi}{2} \cdot(\kappa-1) \cdot T_{m} \cdot \frac{1}{L}
$$

\subsection{ACOUSTIC CONCEPTS}

Some acoustic concepts which are important for proper operation of thermoacoustic will be described. The combination of a plane travelling wave to the right and the reflected wave at the closed end of the tube generate sinusoidal standing wave. The acoustic pressure in the tube is given by [1]:

$$
p_{1}(x)=p_{1} \cdot \cos (k \cdot x)
$$


By integration Newton's second law, the particle velocity is [7]:

$$
u_{1}(x)=\operatorname{Im} \frac{p_{1}}{\rho_{m} \cdot a} \cdot \sin (k \cdot x) .
$$

The position $x$ is measured from the closed end of the resonator, where is a node for velocity and an anti-node for pressure and $k=\omega / a=2 \cdot \pi / \lambda$ is the acoustic wavenumber [11]:

$$
k=\omega / a=2 \cdot \pi / \lambda .
$$

For closed-open tube is $k \cong \pi / 2 \cdot L$ where $\lambda$ and $L$ are the wavelength and the length of the resonator, respectively. The pressure and velocity are spatially $90^{\circ}$ out of phase.

The gas harmonic excursion $\xi_{1}$ is given by $\xi_{1}=u_{1} / \omega$. If we replace $u_{1}$ by its expression from Equation 13 we get:

$$
\xi_{1}=\frac{p_{1}}{\rho_{m} \cdot \omega \cdot a} \cdot \sin (k \cdot x) .
$$

Substituting this result and Equation 3 into Equation 10 and using $a=\sqrt{\kappa \cdot p_{m} / \rho_{m}}$ and $k=\omega / a$ we obtain [7]:

$$
\nabla T_{\text {crit }}=(\kappa-1) \cdot k \cdot T_{m} \cdot \cot (k \cdot x) .
$$

It shows that critical temperature gradient is independent on the acoustic amplitude $p_{1}$ for a given location of the stack center within the standing wave, as we can also see in Equation 11. As the stack approaches the closed end of the resonator, the critical gradient diverges.

\subsection{RATIO OF ACTUAL TEMPERATURE GRADIENT TO THE CRITICAL GRADIENT}

The two modes of operation are characterized in terms of the ratio of the temperature gradient along the stack and the critical temperature gradient [1]:

$$
\Gamma=\frac{\nabla T_{m}}{\nabla T_{\text {crit }}}=\frac{\frac{\Delta T_{m}}{l_{s}}}{\frac{T_{1}}{\xi_{1}}} .
$$

For $\nabla T_{m}>\nabla T_{\text {crit }}, \Gamma>1$ an acoustic power is produced near the channel and the heat flux is toward the pressure node (open end) while for $\nabla T_{m}<\nabla T_{\text {crit }}, \Gamma<1$ an acoustic power is absorbed near the channel and the heat flux is away from the pressure node.

If suitable heat exchangers at temperature $T_{H}$ and $T_{C}$ are installed at the ends of the stack with length of $l_{s}$ this heat flux carries from one exchanger to the other [1]. The temperature difference over the stack is defined as [7]:

$$
\Delta T_{m}=\frac{l_{s} \cdot T_{1}}{\xi_{1}} \cdot \Gamma=T_{H}-T_{C}
$$




\section{THERMOACOUSTIC HEAT FLUX AND ACOUSTIC POWER}

The average values for the heat flux per unit area $\dot{q}$ and the acoustic power per unit volume $\dot{w}$ can be calculated by the expression for the total temperature $T_{1}(y)$ Equation 9 . This average values are proportional to the imaginary part of $T_{1}(y)$ which has a maximum in the distance $\delta_{k}$ perpendicular to the plate and falls to zero at channel surface and at infinity [1]:

$$
\begin{aligned}
& \dot{q}=\frac{1}{2} \cdot \rho_{m} \cdot c_{p} \cdot u_{1} \cdot \operatorname{Im}\left[T_{1}\right], \\
& \dot{\omega}=-\frac{1}{2} \cdot \omega \cdot \beta \cdot p_{1} \cdot \operatorname{Im}\left[T_{1}\right] .
\end{aligned}
$$

The total heat flux along the plate with width of $\Pi$ and gap width $y \approx 2 \cdot \delta_{k}$ was obtained as [1]:

$$
\dot{Q}=-\frac{1}{4} \cdot \Pi \cdot \delta_{k} \cdot T_{m} \cdot \beta \cdot p_{1} \cdot u_{1} \cdot(\Gamma-1),
$$

where $\Gamma=\nabla T_{m} / \nabla \mathrm{T}_{\text {crit }}$, is well known as the ratio of the actual temperature gradient to the critical gradient from Equation 18. The heat flux is proportional to the cross-sectional area $\Pi \cdot \delta_{k}$ and $T_{m} \cdot \beta$ (=1 for ideal gases). Next, it is proportional to the product $p_{1} \cdot u_{1}$ which is zero if the stack is at pressure or velocity node of the standing wave, the maximum value of it is $p_{1}^{2} / 2 \cdot \rho_{m} \cdot a$ and occurs halfway between the node $(L / 2)$. For $\Gamma>1$ the heat flux is toward the pressure node from the heat temperature exchanger to the heat sink.

The total acoustic power is product of integrating the work flux (Equation 21) over the whole space [1]:

$$
\dot{W}=\frac{1}{4} \cdot \Pi \cdot \delta_{k} \cdot l_{s} \cdot \frac{T_{m} \cdot \beta^{2} \cdot \omega}{\rho_{m} \cdot c_{p}} \cdot p_{1}^{2} \cdot(\Gamma-1) .
$$

The acoustic power is proportional to the volume $\Pi \cdot \delta_{x} \cdot l_{s}$ of gas. There can be seen the importance of the ratio $\Gamma$ generating the acoustic power when $\Gamma>1$ or absorbed near the plate when $\Gamma<1$.

The efficiency of the single plate prime mover is defined as ratio Equations 21 and 22 [1]:

$$
\eta=\frac{l_{s} \cdot \beta \cdot \omega \cdot p_{1}}{\rho_{m} \cdot c_{p} \cdot u_{1}} .
$$

Which resembles Equation 10 for the critical temperature gradient then we can write:

$$
\eta=\frac{\nabla T_{c r i t} \cdot l_{s}}{T_{m}}=\frac{\nabla \mathrm{T}_{\mathrm{m}} \cdot l_{\mathrm{s}}}{\Gamma \cdot T_{m}}=\frac{\Delta T_{m}}{\Gamma \cdot T_{m}}=\frac{\eta_{C}}{\Gamma},
$$

where $\eta_{C}$ is Carnot's efficiency the maximum possible efficiency of an engine at $T_{m}$ working between the temperatures $T_{H}$ and $T_{C}$. The efficiency of single-plate prime mover is less than Carnot's efficiency by factor $1 / \eta$ with the limit $\Gamma \rightarrow 1$ where the output power of the device approaches zero for the ideal case. The efficiency is necessarily less than Carnot's for nonzero power. 


\section{ConClusions}

The basic principles of the thermoacoustic effect have been introduced. We primarily focused on understanding of the quarter-wavelength (closed-open) thermoacoustic engine as a prime mover. We showed influence of the main parts of this simple engine as a length of the resonator and a design of stack as a pivotal part of thermoacoustic engine. Thermoacoustic heat engines and refrigerators are an attractive alternative to current conventional system based on its simplicity, reliability and environmentally friendly mode. These initial design calculations provide a basis for further studies, experiments and numerically computations.

\section{ACKNOWLEDGMENTS}

The authors would like to thank the Grant Agency of Czech Republic GACR (project no. P101/11/J019), Grant Agency AS CR (project no. IAA200760801), the Research Centre of MSMT CR (project no. 1M06031) for the opportunity to do research in thermoacoustic and to the company Rauschert, k. s. for provision a suitable material to produce stacks.

\section{REFERENCES}

[1] Swift G.W.: Thermoacoustic Engines, J. Acoust. Soc. Am. 84(4), 1988, 11451180

[2] Swift G.W.: Analysis and Performance of a Large Thermoacoustic Engine, Acoust. Soc. Am. 92(3), 1992, 1551-1563

[3] Petculescu G.: Fundamental Measurements in Standing-Wave and Travelling Wave Thermoacoustic, Dissertation, University of Ohio university, 2002

[4] Garrett S.L.: Thermoacoustic Engines and Refrigerators, The $4^{\text {th }}$ International Meeting of Advances in Thermofluids, Melaka, Malaysia, 1-15

[5] Strutt J.W. (Lord Rayleigh): The Theory of Sound, 2nd edition, Vol. 2, New York, Dover press 1945, 487-491

[6] Rott N.: Thermoacoustic, Advances in Applied Mechanics vol. 20, Academic Press, 1980, 135-175

[7] Tijani M.E.H.: Loudspeaker-Driven Thermo Acoustic Refrigeration, Technische Universiteit Eindhoven, 2001

[8] Ghazali N.M.: Thermoacoustic Heat Engine, Jurnal Teknologi 40(A), University Teknologi Malaysia, 2004, 67-78

[9] Zink F., Vipperman J.S., Schaefer L.A.: Environmental Motivation to Switch to Thermoacoustic Refrigeration, Applied Thermal Engineering 30 , United States, 2010, 119-126

[10] Panhuis P.H.M.W.: Mathematical Aspects of Thermoacoustics, Technische Universiteit Eindhoven, 2009

[11] Nave C.R.: HyperPhysic, Georgia State University, 2011 Available: http://hyperphysics.phy-astr.gsu.edu/hbase/hframe.html 